\title{
Morphological and spectral characteristics of L-band and VHF scintillations and their impact on trans-ionospheric communications
}

\author{
P. V. S. Rama Rao, S. Tulasi Ram, S. Gopi Krishna, K. Niranjan, and D. S. V. V. D. Prasad \\ Space Physics Laboratories, Department of Physics, Andhra University, Visakhaptnam-530 003, India
}

(Received September 8, 2005; Revised February 14, 2006; Accepted February 14, 2004; Online published July 26, 2006)

\begin{abstract}
Amplitude scintillations recorded at 1.5 GHz frequency during the high (1998-1999) and low (2004-2005) sunspot activity periods over a low latitude station, Waltair $\left(17.7^{\circ} \mathrm{N}, 83.3^{\circ} \mathrm{E}\right)$ revealed that the L-band scintillations mostly occur during the post-sunset to midnight hours peaking around 21:00 hr local time with maximum occurrence during equinoxes, moderate during winter and minimum during the summer months. The occurrence, as well as the intensity of scintillations, is found to be strongly dependant on both the season of the year and the sunspot number. Strong (S4-index $>0.45$ ) and fast fading scintillations (fading rates $>40$ fads $/$ min) observed during the post-sunset hours of equinoxes and winter months manifest as several short duration patches at both VHF $(244 \mathrm{MHz})$ and L-band $(1.5 \mathrm{GHz})$ frequencies and are found to be always associated with the range or total Spread-F on ionograms and bubbles/depletions in the Total Electron Content (TEC) measured from a colocated dual frequency GPS receiver, suggesting that these scintillations are of the Plasma Bubble Induced (PBI) type. On the other hand, relatively weak and slow fading scintillations (fading rates $<8$ fads $/ \mathrm{min}$ ) observed around the post-midnight hours of the summer months which appear as long-duration patches ( $>3 \mathrm{hr}$ ) at $244 \mathrm{MHz}$ signal (with practically no scintillation activity at the L-band frequencies) are often found to be associated with frequency Spread-F on ionograms with no depletions in TEC. Further, the presence of Fresnel oscillations observed in the spectrum of $244 \mathrm{MHz}$ suggests that the long-duration scintillations observed are due to the presence of a thin layer of irregularities in the bottom side F-region which are generally known as Bottom Side Sinusoidal (BSS) irregularities. Further, the PBI-type scintillations at L-band frequencies are often found to exceed $10 \mathrm{~dB}$ power levels ( $\mathrm{S} 4>0.45$ ) even during the low sunspot activity period of 2004-2005, and cause Loss of Lock in the GPS receivers resulting in a total interruption in the received signals.
\end{abstract}

Key words: Equatorial ionosphere, ionospheric irregularities, scintillations, fading rate, power spectra, GPS and loss of lock.

\section{Introduction}

Plasma density irregularities in the night time equatorial F-region which affect the radio waves by scattering and diffraction causing scintillations on trans-ionospheric signals, Spread-F on ionograms, plume-like structures in HF/VHF radar maps, and intensity bite-outs in airglow images, are generally known as equatorial Spread-F (ESF) irregularities. Studies on the formation, growth, and dynamics of ESF irregularities using different techniques have been reported in several earlier papers (Calvert and Cohen, 1961; Woodman and LaHoz, 1976; Aarons et al., 1980). Scintillation is the most simple, efficient and less expensive diagnostic technique for the study of ESF irregularities and has been the subject of extensive investigations over the past few decades.

The occurrence of scintillations is highly variable from day-to-day and is controlled by local time, season, solar cycle, latitude, longitude, and geomagnetic activity. Even after several studies on the general morphological features of scintillations (Aarons, 1982) their day-to-day variability is still one of the challenging problems for predicting iono-

Copyright (c) The Society of Geomagnetism and Earth, Planetary and Space Sciences (SGEPSS); The Seismological Society of Japan; The Volcanological Society of Japan; The Geodetic Society of Japan; The Japanese Society for Planetary Sciences; TERRAPUB spheric scintillations, particularly in the equatorial and low latitude regions.

With the increasing applications of Global Positioning Systems (GPS) and satellite-based communication and navigational systems, especially when the millimeter range precision approach is required, as in Satellite Based Augmentations Systems (such as WAAS in the United States and GAGAN in India), the precise occurrence characteristics and prediction of the intensity of scintillations and their effects on L-band frequencies are now very much needed for a better understanding of the space-weather relating to the satellite-based communications.

As the ionosphere over India is situated in one of the highly dynamic regions of the world, covering the geomagnetic equator to the ionization anomaly crest region and beyond, the studies on L-band scintillations and their effects on GPS signals over the Indian sector has drawn considerable attention during recent years. Bandyopadhyay et al. (1997) and Das Gupta et al. (2004) have reported from Calcutta $\left(22.64^{\circ} \mathrm{N}, 88.44^{\circ} \mathrm{E}\right.$ a northern anomaly crest region in India) that the accuracy of position fixing by GPS (L-band frequencies) is considerably degraded during the periods of scintillations during both low and high solar activity periods. Waltair $\left(17.7^{\circ} \mathrm{N}, 83.3^{\circ} \mathrm{E}\right)$ being located in the transition zone between the geomagnetic equator and the ioniza- 
tion anomaly crest regions in India facilitated us to carry out the studies on various scintillation characteristics at L-band frequencies, the results of which are reported and discussed in this paper.

\section{Data and Method of Analysis}

Simultaneous measurements of scintillations at VHF (244 MHz) and L-band (1.5 GHz) frequencies have been carried out from two nearly geostationary satellites, FLEETSAT $\left(73^{\circ} \mathrm{E}\right)$ and INMARSAT $\left(65^{\circ} \mathrm{E}\right)$, respectively, during different phases of the sunspot activity periods of October 1998 to March 1999 (relatively high and ascending phase where the monthly mean Rz values vary between 55.6 and 81.6) and March 2004 to March 2005 (low and descending phase where the monthly mean $\mathrm{Rz}$ values vary between 17.9 and 48.4). A colocated digital ionosonde has also been operated during the same period and the ionograms were obtained at $15 \mathrm{~min}$. intervals. The scintillation data of March 2004-March 2005 has been recorded digitally at a sampling rate of $20 \mathrm{~Hz}$ using an online data acquisition system. The data has been analyzed and different parameters such as percentage occurrences, fading rates, scintillation indices, and power spectra are computed. The data recorded during October 1998 to March 1999 using penchart recorder is scaled carefully and the scintillation index (SI) has been calculated by the third peak method of Whitney et al. (1969), whereas, the scintillation data recorded digitally during March 2004 to March 2005 is used to compute the S4-index which is defined as the square root of the variance of received power divided by the mean value of the received power (Briggs and Parkin, 1963). An empirical relationship between S4 and SI given by Whitney (1974) is used to translate the SI indices into S4 indices so as to compare the intensity levels of scintillations recorded during the two periods.

A dual frequency GPS receiver installed at Waltair under ISRO/GAGAN program was also operated during the same period (2004-2005) to record the scintillations (S4index) at L1 (1575.42 MHz) frequency along with the Total Electron Content (TEC) from the differential phase of the L1 and L2 frequencies. The GPS data from the satellite passes for elevation angles greater than $50^{\circ}$ was considered for the analysis in deriving the S4 index and vertical TEC, to avoid multipath and tropo-scatter effects. In the conversion of vertical TEC, the ionosphere and the protonosphere were assumed as spatially uniform thin shells, and the height of the centroid of ionization of mass distribution was taken as the Ionospheric Pierce Point (IPP). In the present study, the TEC variations during the presence of scintillations at the GPS-L1 frequency of $1.575 \mathrm{GHz}$, the IPP height is taken as 350 km (Davies and Hartmann, 1997; Goodwin et al., 1992; Ciraolo and Spalla, 1997) and the mapping function, assuming the ionosphere as a homogenous thin shell, is used in the conversion of slant TEC to vertical TEC. The bias of the receiver is determined by observing the diurnal variation of TEC at Waltair, and fixing the minimum value by averaging the 3 to $4 \mathrm{hr}$ of data around the day minimum, which is further validated using the bottom-side electron content derived from ionosonde data and topside electron content from the IRI model.

\section{Results}

3.1 Diurnal, Seasonal and Sunspot activity variations of L-band scintillations

The diurnal and seasonal variation in the occurrence characteristics of L-band $(1.5 \mathrm{GHz})$ scintillations for power levels greater than $3 \mathrm{~dB}$ ( $\mathrm{S} 4 \geq 0.15$ ) observed from INMARSAT during the low and descending phase of the sunspot activity period from March 2004 to March 2005 are presented in Fig. 1. It can be observed from this figure that the percentage occurrence of scintillations is predominantly higher during the post-sunset hours and reached a maximum value of 55\% around 21:00 hr local time during March 2004. Further, the percentage occurrence of scintillations is higher during equinoxes, low during winter, and absent during the summer months. During this period (2004-2005), the scintillation occurrence is found to be higher (50\%) during vernal equinox (March, April) of 2004 than during autumnal equinox (September, October) where the percentage occurrence is about $30 \%$ with the peak around 21:00-23:00 hr local time. Also, the percentage occurrence of scintillations is higher in March 2004 $(55 \%)$ where the mean sunspot number $\mathrm{Rz}$ is 49.1 , than in March 2005 (45\%) where the mean sunspot number, Rz reduced to 24.8 evidencing the decrease in scintillation occurrence with the decreasing sunspot number.

With a view to examining the long-term variation in the scintillation activity at L-band frequency with sunspot number, the percentage occurrence of scintillations (SI $\geq 3$ $\mathrm{dB}$ power levels) during the relatively high (the monthly mean $\mathrm{Rz}$ value varies between 55.6 to 81.6) and ascending phase of the sunspot activity period from October 1998 to March 1999, for which data is available, is compared with the percentage occurrence of scintillations during the low (the monthly mean $\mathrm{Rz}$ value varies between 17.9 to 48.4 ) and descending phase of the sunspot activity from October 2004 to March 2005 are presented in Fig. 2. The top panel shows the percentage occurrence of scintillations at $1.5 \mathrm{GHz}$ during the period from October 1998 to March 1999 and the bottom panel shows the occurrence for the period from October 2004 to March 2005. The monthly mean sunspot numbers $(\mathrm{Rz})$ are shown for each month on the $\mathrm{X}$-axis. The numerical values of percentage occurrence of scintillations for each of the months are shown on the top

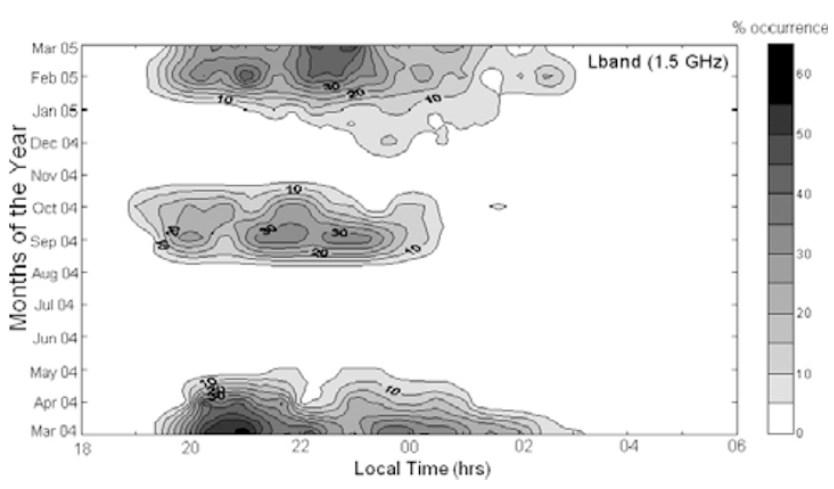

Fig. 1. Contour diagram showing the diurnal and seasonal variation of the percentage occurrence of L-band (INMARSAT) scintillations during the low sunspot year (March 2004-March 2005). 

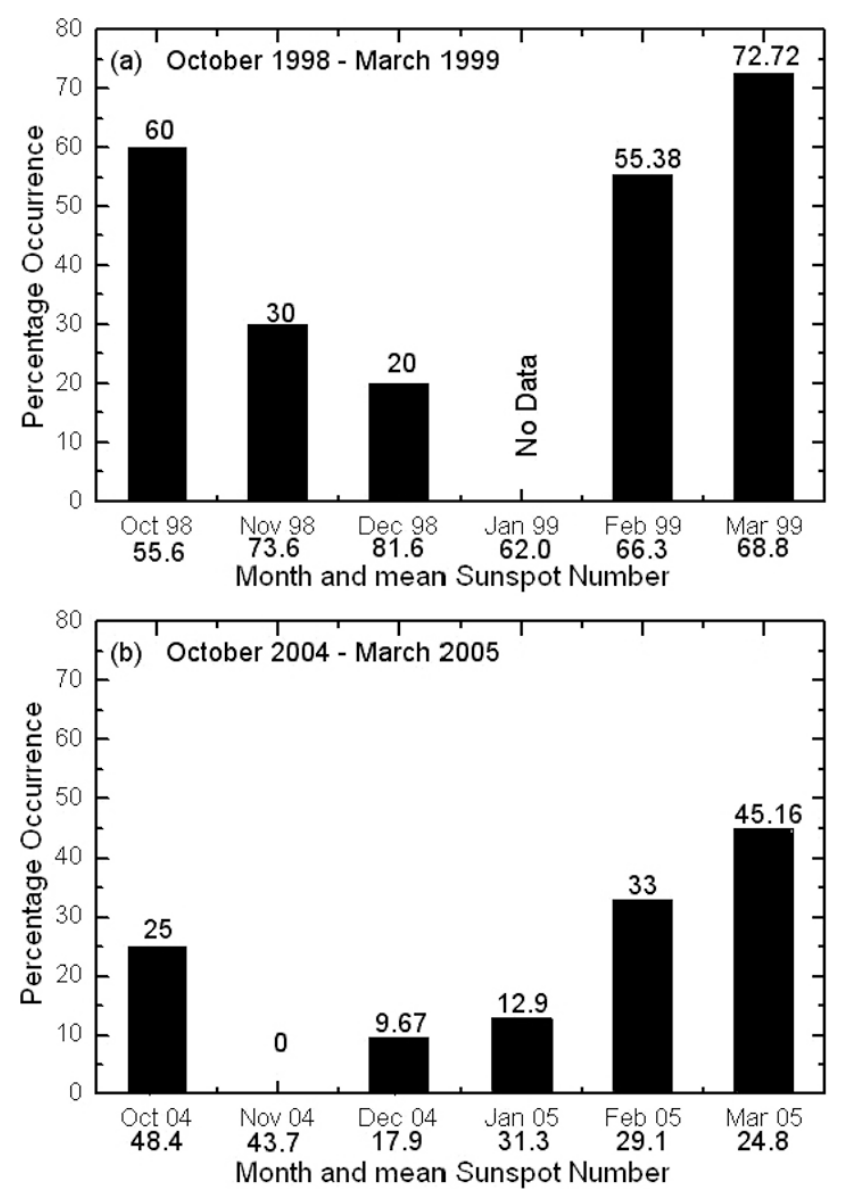

Fig. 2. Percentage occurrence of scintillations $(>3 \mathrm{~dB})$ during (a) high (October 1998-March 1999) and (b) low (October 2004-March 2005) sunspot periods.

of the vertical bars.

It is clear from this figure that during each of the months, the scintillation activity is higher during the high sunspot activity period (October 1998-March 1999) than that of the low sunspot activity period (October 2004-March 2005). Also, it is seen from the figure that the percentage occurrence of scintillation is higher during equinoxial months than during winter months.

It is known that the occurrence of irregularities in the equatorial region increases with the increase of sunspot number, maximizing during the high sunspot activity periods. Also, the occurrence of scintillations is modulated by the seasonal pattern with maximum occurrence during the equinoxes followed by winter months and minimum in summer months. During the high sunspot activity periods, the seasonal modulation in the occurrence of scintillations is masked by the influence of the sunspot number control. However, during relatively moderate to low sunspot number periods, such as 1998-1999 (ascending phase) and 20042005 (low and descending phase), the seasonal control on the scintillation activity is predominantly visible over the sunspot number dependency as may be seen from Fig. 2.

From the present data, it is interesting to note from Fig. 2(a) that, even though the monthly mean sunspot numbers of equinoxial months October $1998(\mathrm{Rz}=55.6)$ and March $1999(\mathrm{Rz}=68.6)$ are lower than that of the winter
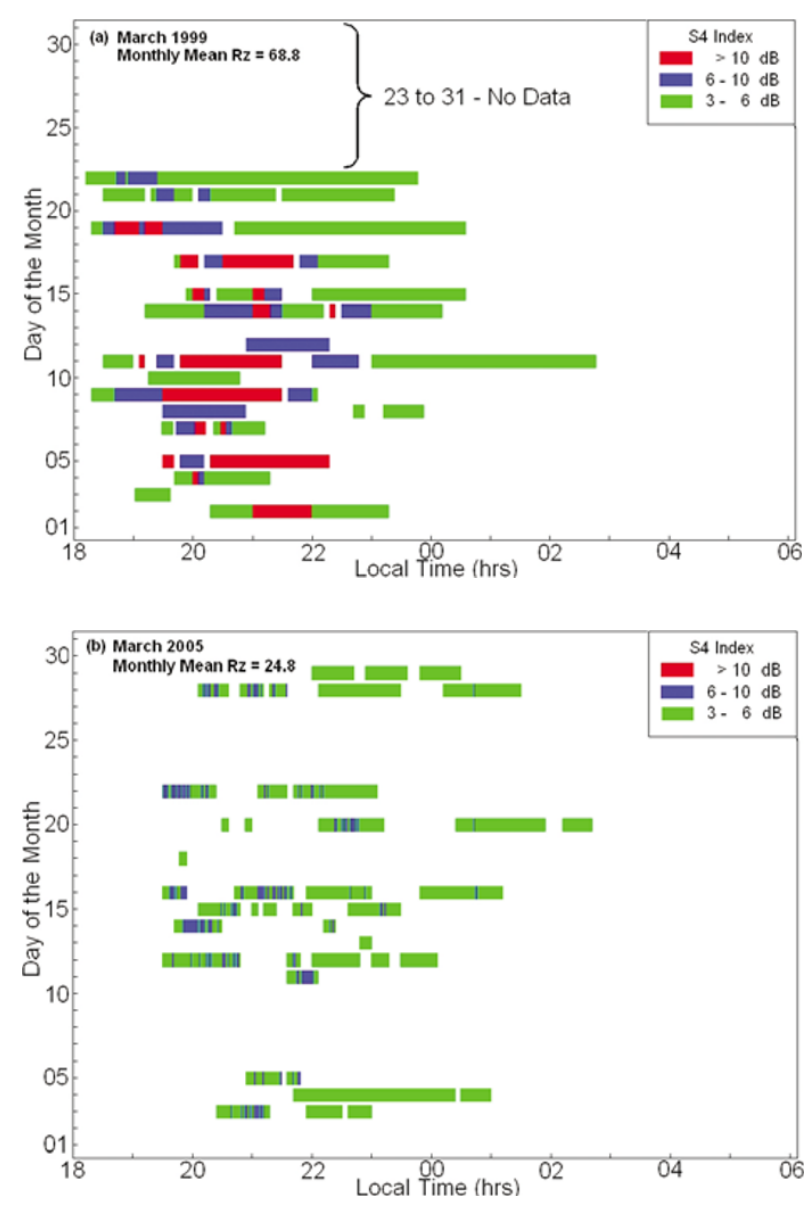

Fig. 3. Diurnal variation of scintillation occurrence at different power (S4) levels on each day during the months of March 1999 and March 2005 representing high and low sunspot years, respectively.

months November $1998(\mathrm{Rz}=73.6)$ and December 1998 $(\mathrm{Rz}=81.6)$, the scintillation activity is much higher in the equinoxes. Also, it can be clearly seen from Fig. 2(b) that the monthly mean sunspot number of the equinoxial month of March $2005(\mathrm{Rz}=24.8)$ is lower than that of the winter month of November $2004(\mathrm{Rz}=43.7)$, but the scintillation activity is much higher in March 2005 (45.16\%) than in November 2004 where the occurrence is zero, suggesting that the seasonal dependence of scintillation occurrence dominates the sunspot number dependency.

Over the sunlit hemisphere, the E-region ionization short circuits the polarization electric fields developed in the Fregion, during the evolution phase of the ESF irregularities, along the field lines. During the equinoxes, the solar terminator aligns symmetrically over the northern and southern hemispheres and thereby simultaneously releasing the E-region conductivity (which acts as a short circuit over sunlit hemisphere) on both the hemispheres causing favorable conditions for the generation of irregularities during the equinoxial months (Yeh and Liu, 1982).

Figure 3 shows the scintillation occurrence at $1.5 \mathrm{GHz}$ on a day-by-day basis for different S4-index (dB) levels during a high sunspot number month of March 1999 (Fig. 3(a)) and a low sunspot number month of March 2005 (Fig. 3(b)). The color-code assigned to the horizontal bars in this figures are such that the green indicates the weak scintillations 
$(3 \mathrm{~dB} \leq \mathrm{S} 4<6 \mathrm{~dB})$, the blue indicates the moderate scintillations $(6 \mathrm{~dB} \leq \mathrm{S} 4<10 \mathrm{~dB})$ and the red indicates strong scintillations ( $\mathrm{S} 4 \geq 10 \mathrm{~dB}$ ). It may be clearly seen from this figure that the occurrence, as well as the intensity of scintillations during the high sunspot activity month of March 1999 (monthly mean $\mathrm{Rz}=68.8$ ), are higher than those during the low sunspot activity month of March 2005 (monthly mean $\mathrm{Rz}=24.8$ ), clearly indicating the effect of sunspot activity on the occurrence of scintillations.

\subsection{VHF and L-band scintillation characteristics}

Simultaneous recording of the scintillations at VHF (244 $\mathrm{MHz}$ ) signal from FLEETSAT and the L-band $(1.5 \mathrm{GHz})$ signal from the INMARSAT during the low sunspot activity period (2004-2005) has enabled us to make a comparative study of the different characteristics of the scintillations with respect to their frequency dependence. The scintillations observed at both frequencies (VHF and Lband) during the post-sunset hours of the equinoxes (March, April, September, and October) and winter months (January, February, November, and December) are generally characterized by several short-duration patches with sudden/abrupt onset, whereas during the summer months the scintillations at the L-band frequency are almost absent during this low sunspot activity year of 2004-2005. But on a few occasions (eight nights), scintillations have been observed around and post-midnight hours of summer solstice (May, June, July, and August) at the VHF frequency of 244 $\mathrm{MHz}$ for long durations. In order to distinguish these two types of scintillations, several scintillation patches observed at VHF and L-band frequencies during this period (March 2004 to March 2005) are carefully examined by evaluating S4-indices, amplitude fading rates, patch durations, power spectral characteristics of scintillations, and their association with Spread-F on ionograms for further investigation.

A total of 183 scintillation patches were observed at 244 $\mathrm{MHz}$ during the post-sunset hours of equinoxes and winter months during the period March 2004-March 2005. In Fig. 4 is shown the histogram of the patch-duration distributions of scintillation patches observed during the post-

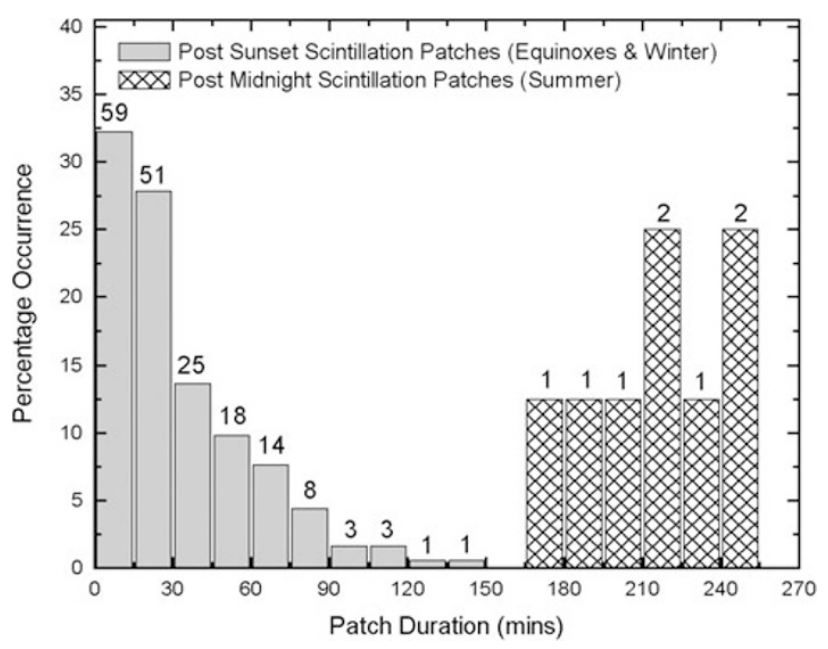

Fig. 4. Histogram showing the patch duration distribution of post-sunset scintillations observed during the equinoxes and winter months, and the post-midnight scintillations observed during the summer months. sunset hours of the equinoxes and winter months and postmidnight hours (eight nights) of the summer months. The numerical values shown on the top of the vertical bars indicate the number of the patches observed in each bin. From this figure, it is observed that the post-sunset scintillation patch durations during the equinoxes and winter months mostly vary from 3 to $90 \mathrm{~min}$, whereas, the post-midnight scintillations observed in the summer months continue to exist for more than $3 \mathrm{hr}$.

In Fig. 5 is shown the $\mathrm{S} 4$ index as a function of fading rate and the association of Spread-F for the corresponding scintillation patches observed at the VHF of $244 \mathrm{MHz}$ during the post-sunset hours of the equinoxes and winter months (Fig. 5(a)), and during the post-midnight hours of the summer months (Fig. 5(b)). The Spread-F observed simultaneously on ionograms is classified as (i) Range Spread F, (ii) Frequency Spread F, and (iii) Total (Range \& Frequency) Spread F.

It may be seen from Fig. 5(a) that the fading rates of these post-sunset scintillations mostly vary from 20 to 45 fades/min and the corresponding S4 indices vary from 0.4 to 0.8 , suggesting that the fast fading rates are often associated with intense scintillations. Also, it can be observed from this figure that these post-sunset scintillations are always
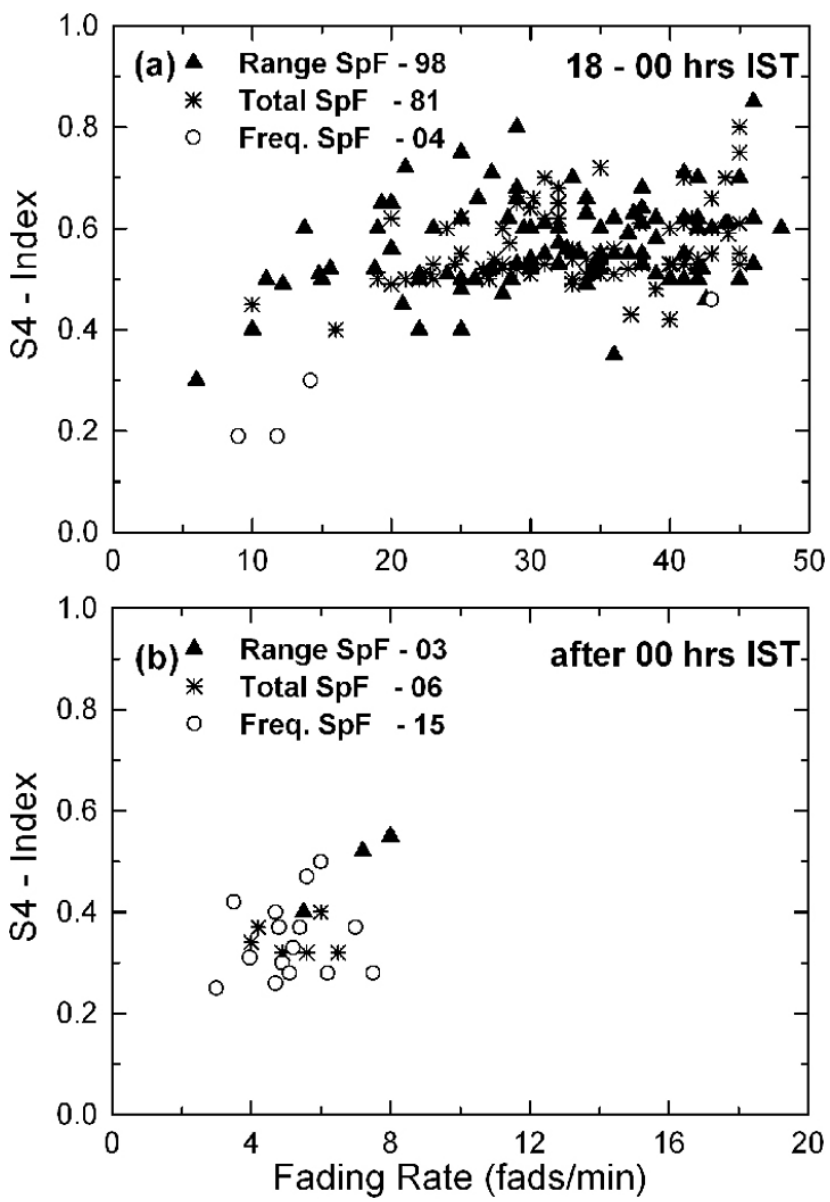

Fig. 5. Scatter plot showing the fading rates, S4 indices and their association with Spread-F on ionograms. (a) scintillation patches observed during the post-sunset hours (18:00-00:00 hr IST) of equinoxial and winter months, (b) scintillation patches observed during the post-midnight hours of (after 00:00 hr IST) of summer months. 

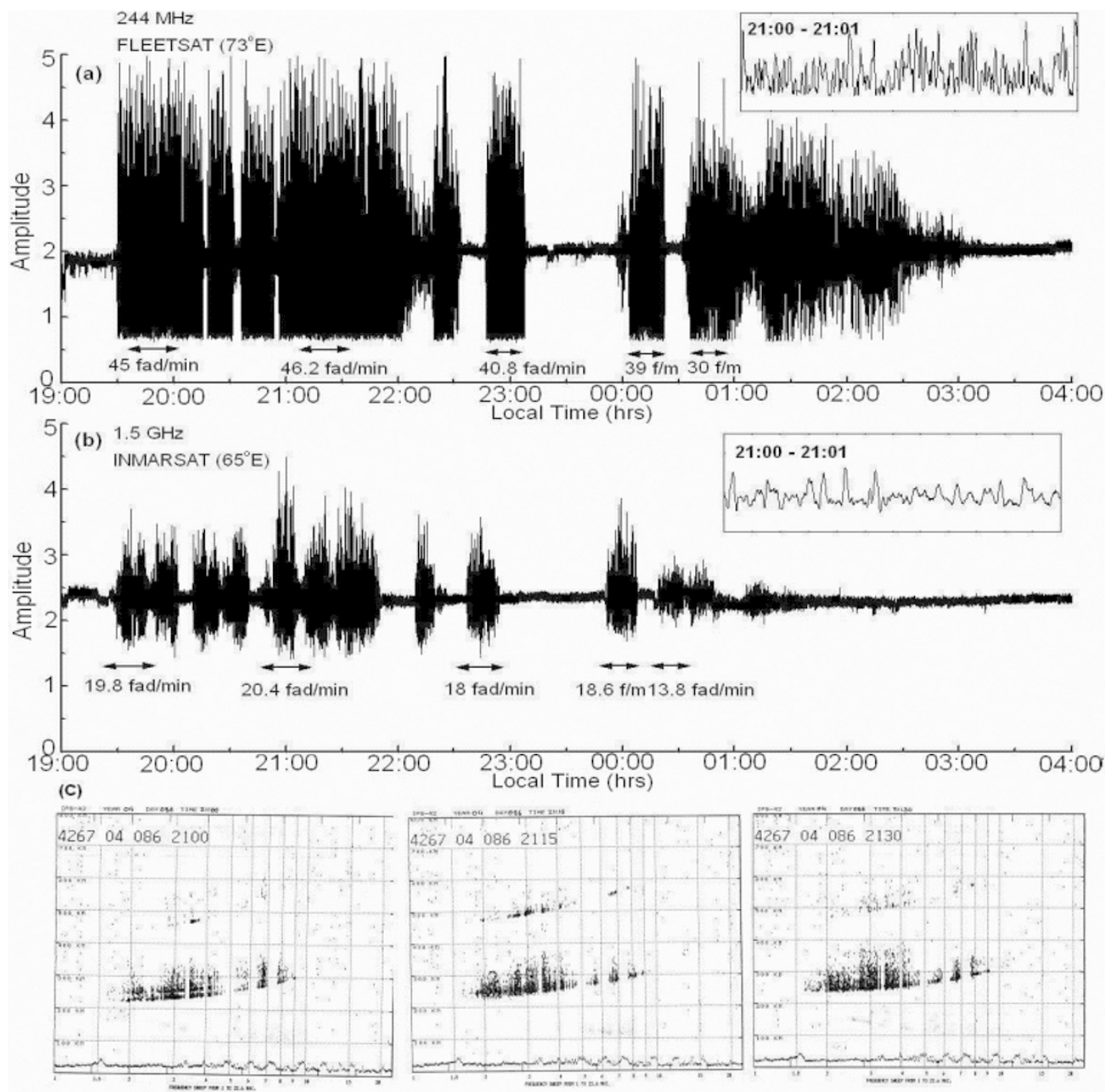

Fig. 6. Typical scintillation records of $244 \mathrm{MHz}$ and $1.5 \mathrm{GHz}$ signals on 26 March 2004 along with the corresponding ionograms showing the presence of range Sp-F.

found to associate with range Spread-F (98 cases) or total Spread-F (81 cases) with a few (four) scintillation events associated with frequency Spread-F which occurred around 23:30 $\mathrm{hr}$ IST and are associated with low fading rates as may be seen from the figure.

But, during the summer months (May, June, July, and August), the scintillation activity is very much reduced. However, on a few occasions (eight nights), long-duration scintillation patches $(>3 \mathrm{hr}$ ) were observed around and after midnight hours at $244 \mathrm{MHz}$ with no scintillations at the Lband frequency of $1.5 \mathrm{GHz}$. The fading rates, S4 indices and their association with Spread-F measured at the beginning, middle, and ending phases of these long-duration scintillations patches and are shown in Fig. 5(b) (with an expanded scale for better visibility). It is noticeable from this figure that the fading rates of these post-midnight scintillations vary from 3 to 8 fades/min and the $\mathrm{S} 4$ index varies from 0.3 to 0.5 . Also, from Fig. 5(b), it can be observed that the post-midnight long-duration scintillation patches are often (15 cases) found to be associated with frequency Spread-F on ionograms with a few exceptions during the early phases of the scintillation events, which are associated with total/range Spread-F.

Figure 6 shows scintillation patches recorded simultaneously both at $244 \mathrm{MHz}$ (panel-a) and at $1.5 \mathrm{GHz}$ (panelb) frequencies on a typical night of 26 March 2004. The measured average fading rates for each of the scintillation patches are indicated with the double-headed arrows on the records for the corresponding durations. The small rectangular boxes in the right-top corners of both the panels show the expanded view of a one-minute scintillation data segment during 21:00 to 21:01 local time. It is very clear from the figure that the fading rates are much higher at VHF (varies between 30 and 46.2 fades/min) than at L-band fre- 

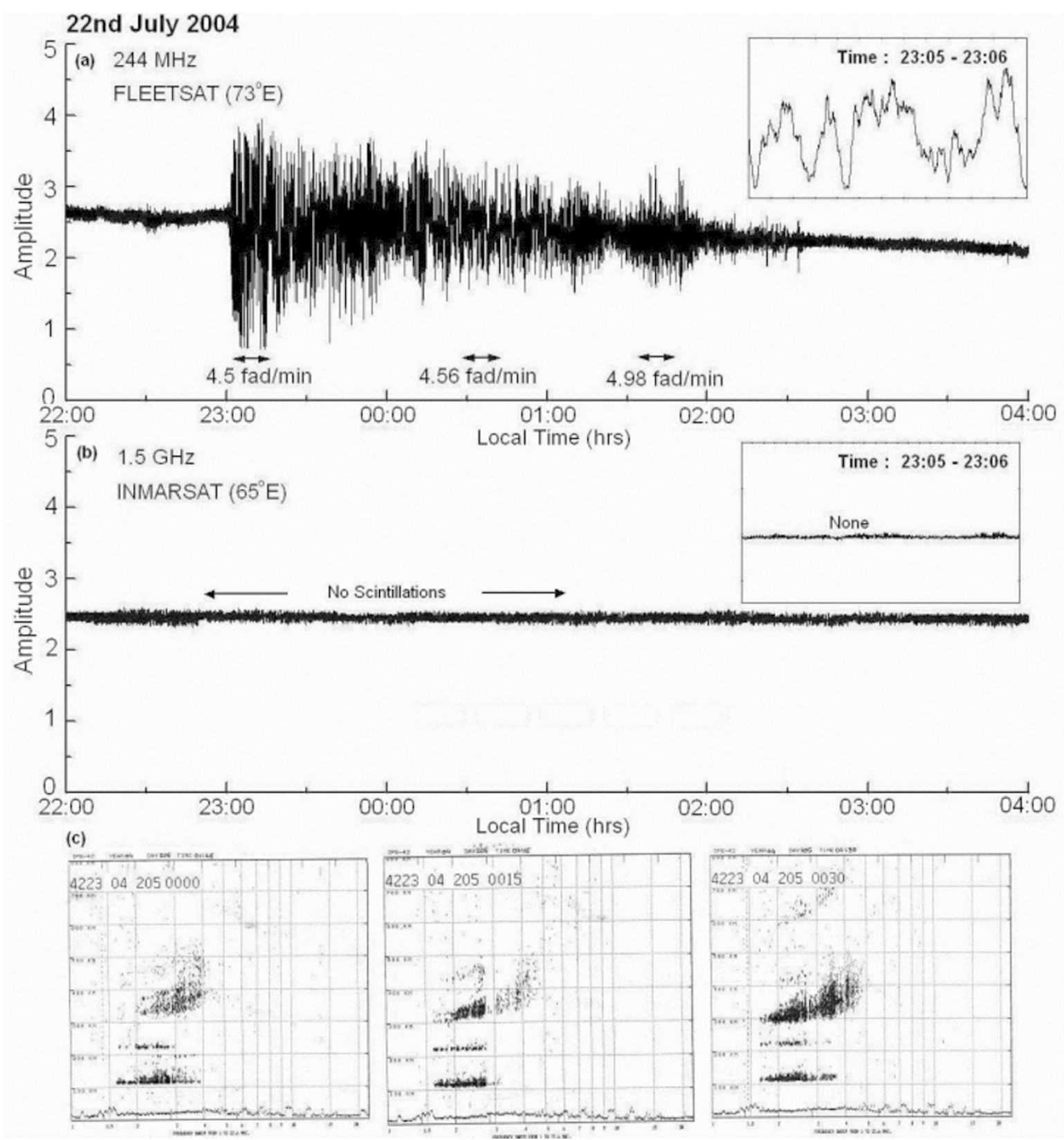

Fig. 7. Typical scintillation records of $244 \mathrm{MHz}$ and $1.5 \mathrm{GHz}$ signals on 22 July 2004 along with the corresponding ionograms showing the presence of frequency Sp-F.

quencies (varies between 13.8 and 20.4 fades/min). The bottom panel (panel-c) shows the presence of range type Spread-F on ionograms during the corresponding times of the scintillation activity on the same night.

Figure 7 shows example of a typical record of a longduration (222 min) late-night scintillation patch at $244 \mathrm{MHz}$ on 22 July 2004 (panel-a) with practically no scintillation activity at the L-band frequency of $1.5 \mathrm{GHz}$ (panel-b). The average fading rates measured at various stages of this scintillation patch are found to vary mostly between 4 and 5 fades/min. The bottom most panel (panel-c) shows a clear association of frequency type of Spread-F on ionograms with this late-night, long-duration scintillation event.

\subsection{Spectral characteristics of VHF Scintillations}

With a view to observe the spatial scale-size distribution of the density irregularities causing the two types of scintil- lations shown in Figs. 6 and 7, power spectra are computed using the FFT algorithm for a two-minute digital data segment of the scintillation patches at $244 \mathrm{MHz}$ recorded at 21:00 hr of 26 March 2004 and at 23:03 hrs of 22 July 2004 and are presented in Fig. 8.

The spectrum of the scintillation patch of 26 March 2004, shown in Fig. 8(a), exhibits a flat response at the low frequency region and steep roll-off in the high frequency region with a break of around $0.8 \mathrm{~Hz}$ suggesting the presence of smaller scale size irregularities. The power law spectral index $(p)$, determined from the slope of the best fit straightline in the frequency range 0.8 to $8 \mathrm{~Hz}$, is found to be -5.86 .

However, the spectrum of 22 July 2004, shown in Fig. 8(b), shows a lower break frequency $\left(f_{B}\right)$ of $0.09 \mathrm{~Hz}$ with a relatively lower spectral slope of -3.04 . Also, the spectrum exhibits a systematic modulation superimposed 

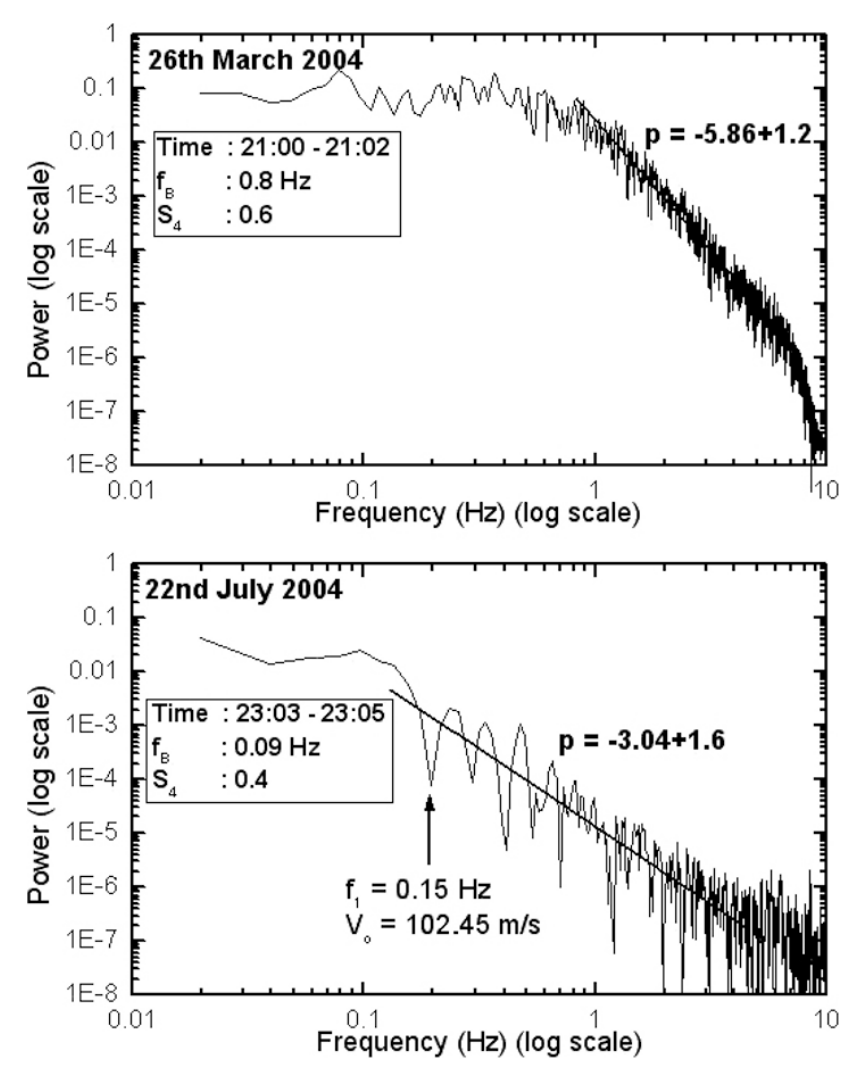

Fig. 8. Power spectrum of 2-min data segments of the scintillation patches observed at $244 \mathrm{MHz}$ shown in Figs. 4 and 5, respectively.

on the linear roll-off portion of the spectrum known as Fresnel oscillations. The first three Fresnel minima are observed at $f_{1}, \sqrt{ } 2 f_{1}, \sqrt{ } 3 f_{1}$, where $f_{1}$ is $0.15 \mathrm{~Hz}$ in this spectrum. The presence of Fresnel oscillations in the spectrum suggests that the irregularities causing these scintillations are confined to a thin layer of thickness, less than $100 \mathrm{~km}$, in the bottom side of the F-region. It was shown by Rufenach (1972) and Yeh and Liu (1982) that the first Fresnel minimum $f_{1}=V_{o} / \sqrt{ }(\lambda Z)$, where $\lambda$ is the wavelength of the signal, $Z$ is the slant range of the observer to the irregularity layer, and $V_{o}$ is the irregularity velocity normal to the propagation path of the satellite signal. In the present case, the slant range $Z$ comes out at $379 \mathrm{~km}$ (assuming the effective height of the ionospheric pierce point (IPP) as 350 $\mathrm{km})$ and the wavelength $\lambda$ at $244 \mathrm{MHz}$ as $1.23 \mathrm{~m}$ which gives rise to a horizontal drift velocity of $102 \mathrm{~m} / \mathrm{s}$. Using this value of horizontal drift velocity, the break frequency ( $\left.f_{B}=0.09 \mathrm{~Hz}\right)$ of Fig. 8(b) corresponds to a spatial scale size of $1138 \mathrm{~m}$, suggesting that the major contribution of forward scattered power is from the irregularities of kilometer scale sizes and a relatively less significant amount of power is contributed from the irregularities of smaller scale sizes.

3.4 L-band scintillation characteristics observed simultaneously from GPS and INMARSAT satellites from Waltair

With the availability of the simultaneous data of S4index of the L-band scintillations from the colocated dual frequency GPS receiver (from orbiting satellites) and the INMARSAT satellites (geostationary) from Waltair, an at-
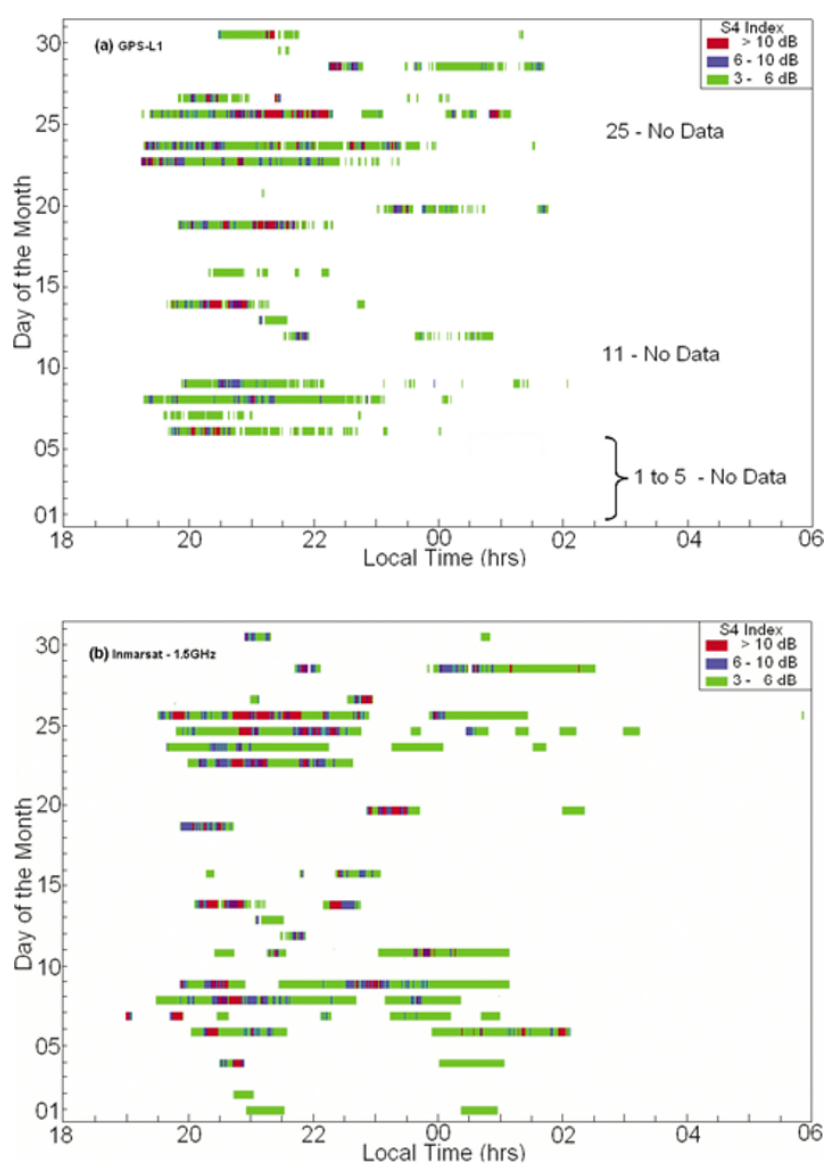

Fig. 9. Bar diagrams of L-band scintillations, at different power (S4) levels, observed from GPS and INMARSAT satellites over Waltair during the month of March 2004.

tempt is made to compare the characteristics of the scintillations from the two different sources. The spatial coverage of the GPS satellites is relatively good and, at any given point of time, a total of about eight satellites are visible from Waltair. The scintillation data (S4-index) recorded at L1 (1575.42 MHz) frequency using dual frequency GPS receiver and those of the L-band $(1.5 \mathrm{GHz})$ scintillations recorded from INMARSAT satellite, broadly show similar morphological features.

In Fig. 9 is shown the occurrence of scintillations for different $\mathrm{S} 4$-index $(\mathrm{dB})$ levels at both the L-band frequencies (1.575 and $1.5 \mathrm{GHz}$ ) on a day-to-day basis for a typical equinoxial month of March 2004 during the low sunspot year of 2004. Figure 9(a) shows the occurrence of scintillations observed from GPS at the L1 frequency of $1.575 \mathrm{GHz}$ whereas in Fig. 9(b) is shown the scintillation occurrence at $1.5 \mathrm{GHz}$ frequency recorded from INMARSAT. It can be seen from the figure that there is a high degree of correspondence in the occurrence features of scintillations observed from both orbiting GPS as well as the geostationary satellite (INMARSAT) signals, particularly during the pre-midnight hours.

3.5 Association of S4-index with depletions in TEC

Further, the scintillations observed in the GPS signals at L1 frequency, shown in Fig. 9(a), are always found to be associated with significant depletions in the Total Electron Content measured from the same GPS satellites visible at 
elevation angles greater than $50^{\circ}$, suggesting that these postsunset scintillations are of the Plasma Bubble Induced (PBI) type (Rama Rao et al., 2004) and are stronger in amplitude. Further, Yeh et al. (1979) and Das Gupta et al. (1983) have also found that TEC-depletions, which are the signatures of plasma bubbles, are always accompanied by an amplitude scintillation of very high intensity and fading rate. They have also reported that during the pre-midnight hours there were occasions when a series of bubbles were observed within a single scintillation patch.

A typical example in the variation of the vertical Total Electron Content (vTEC), derived from GPS, under the strong scintillation conditions of 26 March 2004, as seen from the S4-index is shown in Fig. 10. The solid line represents the variation in the GPS-derived vertical TEC and the dotted line represents the $\mathrm{S} 4$ index variation measured by the GPS receiver at the $\mathrm{L} 1$ frequency. The scale for vertical TEC in TEC units is shown on the left-hand side of the vertical axis and the S4-index is shown on the right-hand side of the vertical axis. It can be observed from this figure that there is a significant depletion in TEC (about 10 TEC units) starting from 19:25 hr local time, which coincides with the onset of scintillations as observed by the sudden increase in the S4-index (dotted line). The depletion in TEC is indicative of a plasma bubble. It may also be noticed here that the scintillations observed at the $1.5 \mathrm{GHz}$ frequency from the geostationary satellite INMARSAT $\left(65^{\circ} \mathrm{E}\right)$ shown in Fig. 6 (panel-b), also starts at the same time. By about 20:00 hr local time, the TEC restores to the normal trend with a value of 52 TEC units, indicating a weakening of the plasma bubble and it is interesting to note that the scintillations at the L1 frequency, as observed from the GPS S4-index (dotted line in Fig. 10) and the scintillations observed at $1.5 \mathrm{GHz}$ frequency observed from the geostationary satellite, INMARSAT (Fig. 6 (panel-b)), have ceased to be present at this local time. Again, another depletion event in TEC observed to start at 20:09 hr local time is also found to be associated with the onset of another scintilla-

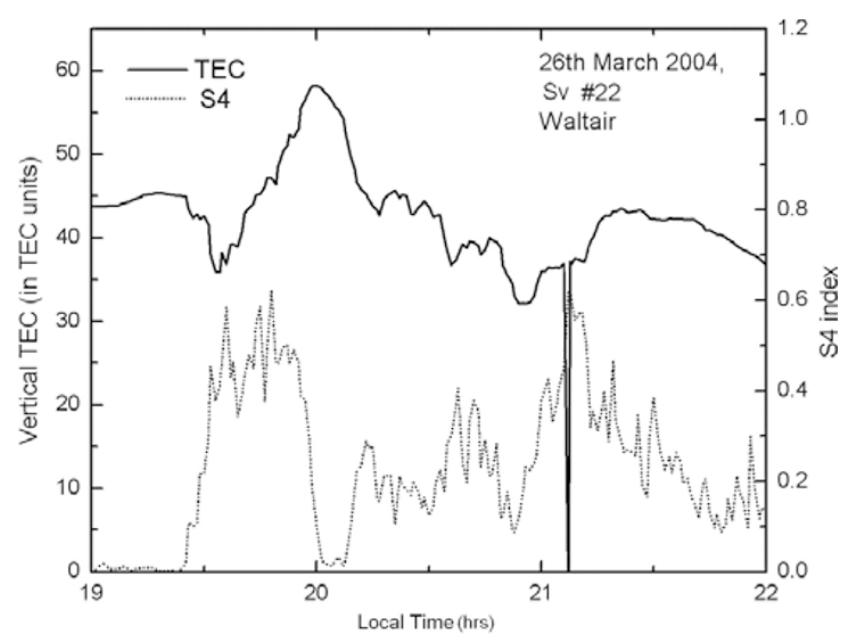

Fig. 10. Variation of L-band scintillation index (S4) observed at L1 frequency from GPS on a typical night of March 26, 2004 and the corresponding variation of Total Electron Content (TEC) derived from the GPS data. tion patch, as observed by both the S4-indices measured from GPS (dotted line in Fig. 10) as well as the geostationary satellite signal (Fig. 6 (panel-b)) at the same time. The TEC value again starts recovering from 21:15 hr local time and the scintillations observed by $\mathrm{S} 4$-index measured from GPS, starts decaying which suggests that these scintillations are associated with the depletions in TEC, which are the signatures of the plasma bubbles.

On the other hand, on 22 July 2004, no significant scintillations and/or TEC depletions are observed from the GPS data, which suggests that the long-duration scintillation patch observed at $244 \mathrm{MHz}$ around the post-midnight hours (Fig. 7 (panel-a)), is not due to plasma bubble-induced irregularities, but could be due to a thin-layer of irregularities in the bottom side of F-region which are generally known as Bottom Side Sinusoidal (BSS) irregularities. Das Gupta et al. (1983) and Basu et al. (1986) have also reported that these BSS-type scintillations generally exist for long durations which maximizes around the post-midnight hours of the local summer and are not associated with any depletions in TEC.

\subsection{Loss of Lock in GPS receivers}

In Fig. 10, is shown the S4-index on the GPS-L1 frequency and the corresponding TEC variations. Around 21:05 hr local time, where the S4 index reaches a value of 0.65 , the TEC suddenly drops to zero, resulting in the loss of lock in the phase channel of the GPS receiver, causing a total interruption in the received signal. However, the receiver regains its phase lock with the signal within a short duration of about 2 to $3 \mathrm{~min}$. It has been reported by Rama Rao et al. (2004) from the data of the S4-index and the vertical TEC obtained from the network of GPS receivers installed at various locations in India, that the scintillations with the S4-index often exceeds 0.45 (power level $>10 \mathrm{~dB}$ ) at the L1-frequency of $1.575 \mathrm{GHz}$ resulting in the Loss of Lock of the GPS receiver for short durations, resulting in interruption in the satellite communication.

\section{Discussion}

During the early evening hours, soon after the sunset when the E-region ionization is quickly eaten away due to the recombination of electrons and ions, the F-region ionization remains relatively high, giving rise to steep vertical gradients in the electron density. Further, the pre-reversal enhancement of the east-ward electric field raises the Flayer at the magnetic equator to high altitudes, where the recombination effects are negligible, creating conditions favorable for the generation of irregularities (Woodman and LaHoz, 1976). Then, through the Rayleigh-Taylor (RT) Instability mechanism, large-scale irregularities (plasmadepleted bubbles) that developed are lifted to the topside of the ionosphere, well above the F-region peak altitude (Kelly, 1989). The polarization electric fields within the bubbles are higher, as a result the bubbles rise to the topside at a velocity much greater than the ambient F-region vertical plasma drift velocity (Anderson and Haerendel, 1979). Steep gradients on the edges of the depletions (Costa and Kelly, 1978) help to generate small-scale irregularities as the bubbles rises to greater heights. These small-scale irregularities in the size range of 100 to $500 \mathrm{~m}$, which are 
known to be responsible for producing strong scintillations at L-band frequencies, co-exist with the kilometer size irregularities causing intense (almost saturated) scintillations with fast fading rates at VHF frequencies.

The co-existence of small-scale irregularities, together with the kilometer size irregularities, causes strong multiple scattering of radio waves, resulting in a range type or total Spread-F on ionograms. Figure 10 further confirms that the scintillations observed on 26 March are associated with TEC depletions, which are the signatures of the plasma bubbles. Das Gupta et al. (1983) have also reported that the scintillations occurring during the post-sunset hours of the equinoxes, which appear as several short-duration patches interspersed with absolutely quiet intervals, are always associated with the depletions in TEC suggesting that these scintillations are induced by Plasma Bubbles.

On the other hand, during the post-midnight hours of summer solstice, the F-region ionization is relatively low and the density gradients are also weak. During these latenight hours the west-ward electric field will not allow the irregularities to grow into plasma-depleted bubbles and are thus confined to thin layers in the bottom side F-region, which manifest in the form of frequency Spread-F on ionograms and cause moderate to intense scintillations at VHF with no scintillations on L-band frequency signals. The smaller value of break frequency $(0.09 \mathrm{~Hz})$ observed in the spectrum shown in Fig. 8(b) suggests that the irregularities of Fresnel scale dimensions contribute the most to the observed scintillations at VHF (Fig. 7(a)). Krishna Moorthy et al. (1979) have also reported that scintillations with slow fading rates (which are termed as Class-II by them) are basically due to irregularities of much larger scale sizes than those responsible for scintillations with fast fading rates (Class-I). These large-scale and relatively weak irregularities are responsible for high fading periods (low fading rates) and low S4-index values in VHF scintillations with practically no scintillation activity at L-band frequencies. Also, the presence of Fresnel oscillations in the roll-off portion of the power spectrum (Fig. 8(b)) indicates that the irregularities are confined to a thin layer of thickness of less than $100 \mathrm{~km}$ (Rufenach, 1972; Yeh and Liu, 1982).

From scintillation measurements at Huancayo $\left(12^{\circ} \mathrm{S}\right)$, Basu et al. (1986) reported that the discrete amplitude scintillation patches observed were associated with plasma bubbles mostly in the post-sunset hours, while continuous scintillation events existing for longer durations were associated with Bottom Side Sinusoidal (BSS) irregularities around post-midnight hours. It has also been reported that the frequency of occurrence of BSS irregularities maximizes in the local summer, as is seen in the present study.

The Plasma Bubble Induced (PBI) scintillations observed during the equinoxial month of March 2004, shown in Fig. 8, 9 exceeds the $10 \mathrm{~dB}$ power level in many cases, and resulted in Loss of Lock of the GPS receiver (Rama Rao et al., 2004), resulting in interruption of the transionospheric signal. It has also been reported earlier that the accuracy of position-fixing by GPS is considerably degraded with an increase in the position dilution of precision (PDOP) value over Calcutta (a northern anomaly crest region over the Indian sector) during the periods of scintillations, even in low solar activity periods (Bandyopadhyay et al., 1997). Further, it is to be expected that the scintillation activity increases substantially during high sunspot activity periods and will adversely effect the trans-ionospheric communications and navigation. Das Gupta et al. (2004) have also shown that, seven or eight GPS/GLONASS satellite links out of 15 satellites simultaneously showed scintillations in excess of $10 \mathrm{~dB}$, which resulted in a position error of $11 \mathrm{~m}$ in latitude and $8 \mathrm{~m}$ in longitude during the high sunspot activity years of 1999-2002.

\section{Summary}

The occurrence of scintillations at L-band frequencies is found to be higher in equinoxes, moderate in winter, and almost absent in summer solstice during this low and descending phase of the sunspot activity period from March 2004 to March 2005. Also, the scintillation activity is found to maximize during the post-sunset hours. The occurrence, as well as the intensity of L-band scintillations, is found to be higher during the high sunspot activity period from October 1998 to March 1999 than during the low sunspot activity period from October 2004 to March 2005 suggesting that the scintillation activity is strongly dependant on the sunspot number. It was also found that the seasonal dependence of scintillation occurrence is more dominant than that of the sunspot number dependency.

The scintillations observed during the post-sunset hours of the equinoxes and winter months are found to be intense with high fading rates, large S4-indices, and appear as several short-duration patches at both VHF and L-band frequencies. These intense and fast scintillations are always found to be associated with range or total Spread-F on ionograms and depletions/bubbles in TEC measured from the colocated dual frequency GPS receiver, suggesting that these scintillations are of the Plasma Bubble Induced (PBI) type. The spectrum of these Plasma Bubble Induced scintillations at $244 \mathrm{MHz}$ frequency reveals that the smaller scale size irregularities co-exist with the kilometer scale size irregularities causing intense (almost saturated) scintillations at VHF and moderate to intense scintillations at L-band frequencies.

During the summer solstice, no significant scintillations are observed both on the GPS and INMARSAT signals at L-band frequencies. On the other hand, on a few occasions, strong to moderate scintillations were observed at VHF around the post-midnight hours of the summer solstice. These scintillations are characterized by longduration patches ( 3 to $4 \mathrm{hr}$ ) with slow fading rates ( 3 to 8 fades/min) and are often found to be associated with the frequency Spread-F on ionograms. No depletions in TEC were found to be associated with these long-duration scintillations during the summer solstice months. Further, the presence of Fresnel oscillations in the spectrum of these scintillations at $244 \mathrm{MHz}$ suggests that the irregularities causing scintillations are confined to a thin layer of thickness of less than $100 \mathrm{~km}$ in the bottom side of F-region, generally known as Bottom Side Sinusoidal (BSS) irregularities (Rufenach, 1972; Yeh and Liu, 1982; Bhattacharya et al., 2001).

The Plasma Bubble Induced scintillations at L-band fre- 
quencies during the post-sunset hours of the equinoxes and winter often exceed the $10 \mathrm{~dB}$ power levels $(\mathrm{S} 4>0.45)$, which may cause Loss of Lock in the GPS receivers and consequently create an interruption in the received signal, even during the low sunspot activity periods. Further, the scintillations activity is expected to increase substantially during the high sunspot activity periods and is likely to increase the Loss of Lock events and adversely affect the trans-ionospheric communications and satellite navigation systems.

Acknowledgments. The authors wish to express their sincere thanks to Dr. B. V. Krishna Moorthy for kindly going through this manuscript and making valuable suggestions. One of the authors (P. V. S.) wishes to express his sincere thanks to CSIR for providing him with an Emeritus Scientist program. S. T. R. wishes to express his grateful thanks to DST for providing him with a JRF in the project to carry out a part of this work.

\section{References}

Aarons, J., Global morphology of ionospheric scintillation, Proc. IEEE, 70, 360-378, 1982.

Aarons, J., J. P. Mullen, H. E. Whitney, and E. M. Mackenzie, The Dynamics of Equatorial Irregularity Patch Formation, Motion and Decay, J. Geophys. Res., 85, 139-149, 1980.

Anderson, D. N. and G. Haerendel, The motion of depleted plasma regions in the equatorial ionosphere, J. Geophys. Res., 16, 939-945, 1979.

Bandhyopadhyay, T., A. Guha, A. Das Gupta, P. Banerjee, and A. Bose, Degradation of navigational accuracy with Global Positioning System during periods of scintillation at equatorial latitudes, Electron. Lett., 33, 1010-1011, 1997.

Basu, S., Su. Basu, C. E. Valladares, A. Das Gupta, and H. E. Whitney, Scintillations associated with bottomside sinusoidal irregularities in the equatorial F-region, J. Geophys. Res., 91, 270-276, 1986.

Bhattacharyya, A., S. Basu, K. M. Groves, C. E. Valladares, and R. Sheehan, Dynamics of equatorial $F$ region irregularities from spaced receiver scintillation observations, Geophys. Res. Lett., 28, 119-122, 2001.

Briggs, B. H. and I. A. Parkin, On the variation of radio star and satellite scintillations with zenith angle, J. Atmos. Terr. Phys., 25, 339-365, 1963.

Calvert, W. and R. Cohen, The interpretation and synthesis of certain Spread-F configuration appearing on equatorial ionograms, J. Geophys. Res., 66, 3125, 1961.

Ciraolo, L. and P. Spalla, Comparison of ionospheric total electron content from the Navigation Satellite System and the GPS, Radio Sci., 32, 10711080, 1997.

Costa, E. and M. C. Kelly, On the role of steepened structures and drift waves in equatorial Spread-F, J. Geophys. Res., 83, 4359-4364, 1978.

Das Gupta, A., Basu, S., J. Aarons, J. A. Klobuchar, Basu, Su., and A. Bushby, VHF amplitude scintillations and associated electron content depletions as observed at Arequipa, Peru, J. Atmos. Terr. Phys., 45, 1526, 1983.

Das Gupta, A., S. Rat, A. Paul, P. Banerjee, and A. Bose, Errors in position-fixing by GPS in an environment of strong equatorial scintillations in the Indian zone, Radio Sci., 39, (RS1S30), doi:10.1029/ 2002RS002822, 2004

Davies, K. and G. K. Hartmann, Studying the ionosphere with Global Positioning System, Radio Sci., 32, 1695-1703, 1997.

Goodwin, G. L., J. H. Silby, K. J. W. Lynn, A. M. Breed, and E. A. Essex, Total electron content and ionospheric slab thickness measurements using GPS satellites, Proc. South Pacific Solar-Terrestrial Physics Workshop, Tenth National Congress of the Australian Institute of Physics, Univ. of Melbourne, Melbourne, Australia, 1992.

Kelley, M. C., The Earth's Ionosphere, Academic Press, San Diego, 1989.

Krishna Moorthy, K., C. Ragha Reddi, and B. V. Krishna Murthy, Nighttime ionospheric scintillations at the magnetic equator, J. Atmos. Terr. Phys., 41, 123-134, 1979.

Rama Rao, P. V. S., K. Niranjan, D. S. V. V. D. Prasad, S. Gopi Krishna, and S. Tulasi Ram, Simultaneous Observations of VHF and L-band Scintillations from an Indian Low Latitude Station, Waltair $\left(17.7^{\circ} \mathrm{N}\right.$, 83.3 ${ }^{\circ}$ E), Proc. of IBSS-2004, Trieste, Italy, 2004.

Rufenach, C. L., Power law wave number spectrum deduced from ionospheric scintillation observations, J. Geophys. Res., 77, 4761-4772, 1972.

Whitney, H. E., Notes on the relationship of scintillation index to probability distribution and their uses for system design, Rep. AFCRL-TR-740004, Air Force Cambridge Res. Lab., Hanscom Air Forse Base, Mass, 1974.

Whitney, H. E., J. Aarons, and C. Malik, A proposed index for measuring ionospheric scintillations, Planet. Space Sci., 17, 1069-1073, 1969.

Woodman, R. F. and C. Lahoz, Radar observations of F-region equatorial irregularities, J. Geophys. Res., 81, 5447-5466, 1976.

Yeh, K. C. and C. H. Liu, Radio wave scintillations in the ionosphere, Proc. IEEE, 70, 324-360, 1982.

Yeh, K. C., H. Soicher, C. H. Liu, and E. Borelli, Ionospheric bubbles observed by the Faraday rotation method at Natal, Brazil, Geophys. Res. Lett., 6, 473-475, 1979.

P. V. S. Rama Rao (e-mail: palurirao@yahoo.com), S. Tulasi Ram, S. Gopi Krishna, K. Niranjan, and D. S. V. V. D. Prasad 